\title{
Association of physical inactivity with circulatory disease events and hospital treatment costs
}

This article was published in the following Dove Press journal:

Clinical Epidemiology

16 April 2013

Number of times this article has been viewed

\author{
Rachel C Davey' \\ Thomas Cochrane ${ }^{1,2}$ \\ 'Centre for Research and Action in \\ Public Health, University of Canberra, \\ ACT, Australia; ${ }^{2}$ Centre for Sport \\ and Exercise Research, Staffordshire \\ University, Stoke on Trent, United \\ Kingdom
}

Purpose: Epidemiological studies of chronic disorders need to consider more responsive outcomes, particularly those that manifest themselves across a defined population over a shorter time period, to improve our ability to detect the causes of and intervene in the global epidemics of today. We explore the use of hospital episode statistics as a candidate for this role and estimate the strength of the association of circulatory disease-related events with physical inactivity, considered here as an undesirable health behavior.

Settings, patients, and methods: The primary research was set in a mid-sized city in central England. Aggregation was at output area level (comprising 300 residents); 51 of which were included. A random sample of 761 adults was selected to obtain estimates of the mean level of physical activity within each area. Circulatory disease hospital events were recorded and aggregated by output area over a 2-year period. Hierarchical linear modeling was used to establish the strength of the association between area-level physical activity and circulatory disease events. Sex, age, and reporting quarter were included as additional individual-level explanatory variables.

Results: Areas reporting greater activity were less likely (event rate ratio $=0.855 ; 95 \%$ confidence interval [CI]: $0.78-0.94)$ to have a circulatory disease event, as were females (0.593; 95\% CI: $0.47-0.75)$. Areas with older residents $(1.578 ; 95 \%$ CI: $1.5-1.66)$ and later reporting quarters $(1.095 ; 95 \% \mathrm{CI}: 1.04-1.15)$ were more likely to report circulatory disease events.

Conclusion: This study supports the use of hospital episode statistics as an outcome measure in the epidemiology of circulatory disease and reaffirms the potential importance of physical inactivity in the disease process.

Keywords: exercise, cardiovascular disease, lifestyle, physical environment, chronic disease epidemiology

\section{Introduction}

Since the pioneering research of Morris and colleagues in the UK in the 1950s and Paffenbarger and Hale in the US in the 1970s, ${ }^{1,2}$ physical inactivity has become established as an important modifiable risk factor in a number of chronic diseases..$^{3-5}$ Much of the literature has relied on coronary heart disease mortality or all-cause mortality as the primary outcome measure to establish the link. Whilst this has been undoubtedly seminal research, the end-point, mortality, requires a long wait. It is also extremely difficult to obtain good estimates of the strength of the relationship, as it is usually necessary to observe or, at least, categorize habitual physical activity over a long period. Furthermore, it is difficult to obtain reliable estimates of potential health care cost-benefits of interventions designed to increase physical activity in hospital populations.
Correspondence: Thomas Cochrane Professor of Sport, Health and Exercise, Centre for Research and Action in Public Health, University of Canberra,

ACT 260I, Australia

Tel +61262015359

Fax +61262015403

Email tom.cochrane@canberra.edu.au 
A way around both of these challenges might be to explore the relationship between physical activity and health-related outcomes that may be more responsive, ie, manifest themselves across a defined population over a shorter time period and/or be more sensitive to variation in physical activity, and which may be valued reliably in terms of costs to the health care sector. One source of routinely collected data that may be a candidate for such a role could be hospital episode statistics. We report a pilot study carried out across a single city in the West Midlands region of the UK to test this thesis and to establish estimates of the strength of the association between physical activity and directly measured and valued health outcomes.

We focus our attention on circulatory disease events since these outcomes have well-established links with physical inactivity and at least some of the multifactorial mechanisms for benefit are known. ${ }^{6}$

\section{Methods}

\section{Small area analysis of hospital episodes}

The purpose of the study was to examine whether physical activity, measured at the areal level, was associated with health care treatment episodes for circulatory disorders.

\section{Setting}

The research was set in a mid-sized city (population 250,000) in the West Midlands region of England and the focus was on relatively deprived inner-city areas.

\section{Study areas}

The geographical unit selected to determine study areas was the lower level super output area (LSOA). This is the smallest unit for which population census data are made available in the UK. In England, there are 32,482 LSOAs, which have an average population of 1,500 and a minimum population of 1,000. Each LSOA is made up of 4-6 output areas (OA) and the latter was chosen as the level of geographical aggregation for this analysis. After ranking into deciles of deprivation (based on the English Indices of Multiple Deprivation 2004), ${ }^{7}$ and excluding areas adjacent to the city boundaries and areas undergoing significant redevelopment, ten LSOAs were selected randomly from the six deciles of highest deprivation, yielding 51 OA for inclusion in the analysis.

\section{Primary measures}

Area-level physical activity

Estimates of physical activity for each output area included in the study required a random sample survey of residents from the selected areas. A random probability sample of addresses in the selected LSOAs was chosen. A sample size requirement of 600 , with approximately 60 in each area, was calculated to provide adequate precision (to within 5\% with $95 \%$ CI for a total target population $\sim 15,000$ ) for population proportion estimates. The survey was undertaken independently by the National Centre for Social Research (NatCen, London, UK). Full details of the sample and survey processes are available in our earlier publication and the technical report. ${ }^{8,9}$ Physical activity was estimated using the International Physical Activity Questionnaire (Long version), ${ }^{10}$ which provides estimates of weekly energy expenditure (in MET minutes/ week, where the MET or metabolic equivalent of an activity expresses the energy cost of the activity as multiples of the resting metabolic rate) in four activity domains: workrelated, active transport, garden and domestic, and leisure. The questionnaire was administered by computer-assisted personal interview.

\section{Circulatory disease events}

Circulatory disease events (International Statistical Classification of Disease and Related Health Problems, 10th Revision ${ }^{11}$ codes I00-I99) were recorded over a 2-year period from January 1, 2006 to December 31, 2007 for each of the $51 \mathrm{OA}$.

\section{Ethical considerations}

This research was conducted according to the ethical principles laid out in the Declaration of Helsinki and was approved by the Institutional Research Ethics Committee. All participants in the physical activity survey gave informed consent to take part, following receipt of written (letter and leaflet in advance) and verbal information (at interview) describing the study. Access to anonymized information relating to hospital episodes was granted by the Information Governance Manager, Directorate of Information Management and Technology of the local Primary Care Trust following confirmation of compliance with both the Data Protection Act 1998 and the National Health Service Confidentiality Code of Practice.

\section{Analysis strategy Variables available}

Sex, age, ethnicity, deprivation decile, and time of year (reporting quarter) were available as well as the primary diagnosis for each hospital episode considered. Thus, it was possible to test and adjust for these factors within the analyses. The mean physical activity score for 
each OA was also calculated to give an estimate of physical activity. In addition, the cost of each hospital episode could be valued through the associated Healthcare Resource Group code and indicative cost for the procedure, allowing estimates to be obtained of the association of physical inactivity with costs for treating hospital episodes for circulatory disorders. All costs reported here are based on unadjusted cost estimates from 2007.

\section{Explanatory models}

Circulatory disease episodes were considered to be rare events that were recorded as counts over a set period, 2 years in this instance (individual patients could have more than one circulatory disease event during the recording period). Thus, the Poisson model with equal exposure was considered a reasonable model for these events. Sex, age, ethnicity, and time of year (quarter) were treated as potential individual level variables, while deprivation decile and mean physical activity for each area were considered as potential area-level variables. A hierarchical linear modeling approach, using the Poisson model with equal exposure and a logarithmic link function, was used to model these events. ${ }^{12}$

To illustrate the potential link between physical inactivity and treatment costs, combined costs for circulatory disease treatments were simply plotted against area-level physical activity. Bootstrap resampling, with 1,000 repetitions, was used to check the sensitivity of this analysis to high-cost events.

\section{Model development}

For a Poisson model, the number of events recorded for individual $i$ in neighborhood $j$ is a function of the time interval over which exposure occurs and the event rate $\lambda_{i j}$. The basic model has the form:

Link function

$$
\eta_{i j}=\log \left(\lambda_{i j}\right)
$$

Individual-level model

$$
\eta_{i j}=\beta_{0 j}+\beta_{1 j} X_{1 i j}+\ldots \beta_{k j} X_{k j i}
$$

where $\beta_{0 j}$ is the intercept term for neighborhood $j, \mathrm{X}_{\mathrm{k} i j}$ is the individual level factor $\mathrm{k}$ for participant $i$ in neighborhood $j$ and $\beta_{\mathrm{k} j}$ is the regression coefficient for factor $\mathrm{k}$ in neighborhood $j$.

Area-level model: At the area level, the coefficients $\beta_{\mathrm{k} j}$ in equation 1 are modeled as linear combinations of an intercept term, $\gamma_{\mathrm{k} 0}$, together with the area-level factors, $\mathrm{W}_{\mathrm{s} j}$, and a random effects term, $\mathrm{u}_{\mathrm{kj}}$, as follows:

$$
\beta_{\mathrm{k} j}=\gamma_{\mathrm{k} 0}+\gamma_{\mathrm{k} 1} \mathrm{~W}_{1 j}+\ldots+\gamma_{\mathrm{ks}} \mathrm{W}_{\mathrm{s} j}+\mathrm{u}_{\mathrm{k} j}
$$

where $\gamma_{\mathrm{ks}}$ is the regression coefficient for area-level factor $\mathrm{W}_{\mathrm{s}}$ in area $j$ and $\mathrm{s}$ is the number of area-level factors included in the model.

Equation 2 allows for each individual level coefficient to be modeled either in fixed, nonrandomly varying, randomly varying, or a combination of randomly and nonrandomly varying forms. Building of the multilevel model was rather complex. Our approach, for each separate potential area-level index, was to use the nonrandomly varying form of equation 2 $\left(\mathrm{u}_{\mathrm{k} j}\right.$ set to 0$)$ as a starting iteration. Level 2 coefficients, $\gamma_{\mathrm{ks}}$, which made significant $(P<0.05)$ explanatory contributions to the variation in the model were retained and further iterations were carried out by adding a randomly varying component $\left(\mathrm{u}_{\mathrm{k} j}\right)$ to test if this significantly improved the model fit. Finally, these separate level 2 models were combined into an overall best-fit multilevel linear regression model, again by retaining only those coefficients making a significant $(P<0.05)$ contribution to the overall model fit. All analyses were carried out using HLM-6 (Scientific Software International, Lincolnwood, IL, USA) using level 1 and level 2 data files created with SPSS software (version 16; IBM, Armonk, NY, USA).

Weighting of area-level data: Weights were applied at the area level to account for population differences in each area. The weighting variable was the estimated residential population in each area, calculated from the average count per residence in each selected LSOA (total population/number of residential addresses) times the number of residences in each output area.

Transformation of variables: The distribution of physical activity scores in the small area sample was positively skewed. Thus, it was necessary to transform this variable (using the square root transformation) for use in the analysis. In addition, we wanted the primary outcomes, event rate ratios, to be provided for meaningful changes in the main factors under consideration. Age was transformed into age decades (division by 10 years) and the transformed physical activity variable was used in its standard normal form (subtract mean from each value and divide by the standard deviation [SD]). Event rate comparisons, therefore, are provided for decade changes in age and a one-standard-deviation change in physical activity. 


\section{Results}

\section{Characteristics of sample populations}

The characteristics of the 761 respondents who took part in the survey to estimate area-level physical activity and the 3,115 patients aged over 16 years who had one or more hospital episode(s) during the 2-year evaluation period are shown in Table 1 . The age and ethnicity distributions of the samples are similar. Ethnic diversity is low in the city as a whole so categories other than white British have been collapsed into a single category. The patient population has a larger proportion of females, which is probably because this population includes women of childbearing age, who tend naturally to require more hospital care. The distribution among deciles 4-6 (lower deprivation) differs slightly between the two samples but otherwise the two populations have broadly similar characteristics. Also shown in Table 1 for comparison are the mean numbers of circulatory events for each demographic category.

The mean physical activity for each of the 51 OA is illustrated in Figure 1. Overall, the median number of years for which survey respondents had lived in their area was 16 years.

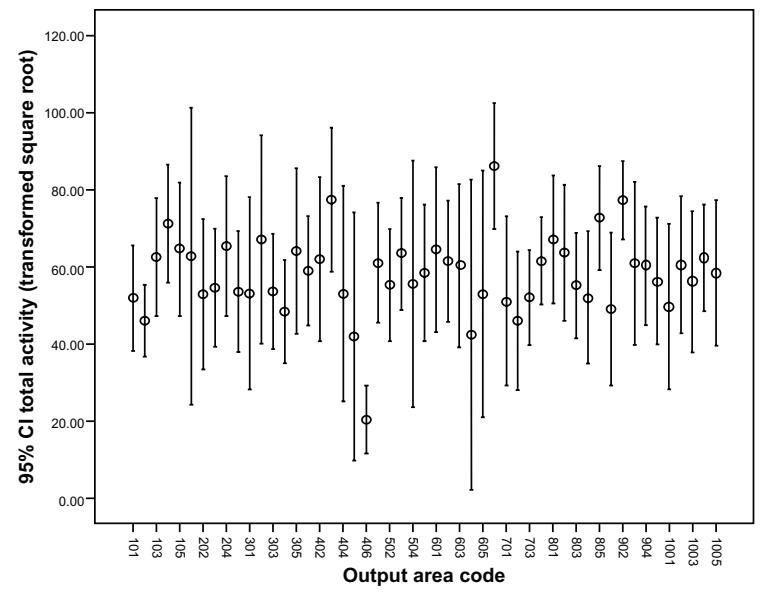

Figure I Error bar plot of square root transformed total activity by output area. Note: Error bars represent $95 \%$ confidence interval for the sampled means.

Within areas, the median number of years lived in the area ranged between 4.5 and 32 years, which confirms that our sample, as intended, was drawn from established communities. The number of respondents in each area sample ranged from 5 to 26 , with a mean of 15 . The intercluster correlation coefficient

Table I Sample characteristics and summary of circulatory disease events by demographic category

\begin{tabular}{|c|c|c|c|c|c|c|}
\hline & \multicolumn{4}{|c|}{ Population sample } & \multirow{3}{*}{$\begin{array}{l}\text { Mean CD } \\
\text { events }\end{array}$} & \multirow[t]{3}{*}{$P$ value } \\
\hline & \multicolumn{2}{|c|}{ Physical activity estimates } & \multicolumn{2}{|c|}{ Hospital episodes } & & \\
\hline & $\mathbf{N}$ & $\%$ & $\mathbf{N}$ & $\%$ & & \\
\hline \multicolumn{7}{|l|}{ Sex } \\
\hline Male & 343 & 45.1 & 1162 & 37.3 & 0.27 & $<0.001$ \\
\hline Female & 418 & 54.9 & 1953 & 62.7 & 0.12 & \\
\hline \multicolumn{7}{|l|}{ Age group } \\
\hline $16-24$ & 76 & 10.0 & 441 & 14.2 & 0.01 & 0.001 \\
\hline $25-44$ & 276 & 36.3 & 1055 & 33.9 & 0.05 & \\
\hline $45-64$ & 235 & 30.9 & 814 & 26.1 & 0.22 & \\
\hline $65+$ & 174 & 22.9 & 805 & 25.8 & 0.40 & \\
\hline \multicolumn{7}{|l|}{ Ethnicity } \\
\hline White British & 699 & 91.9 & 2808 & 90.1 & 0.18 & 0.17 \\
\hline All other ethnic groups & 62 & 8.1 & 307 & 9.9 & 0.11 & \\
\hline \multicolumn{7}{|l|}{ Deprivation decile ${ }^{\mathrm{b}}$} \\
\hline I most deprived & 178 & 23.4 & 685 & 22.0 & 0.24 & $<0.001$ \\
\hline 2 & 132 & 17.3 & 703 & 22.6 & 0.15 & \\
\hline 3 & 142 & 18.7 & 598 & 19.2 & 0.21 & \\
\hline 4 & 75 & 9.9 & 605 & 19.4 & 0.14 & \\
\hline 5 & 75 & 9.9 & 232 & 7.4 & 0.11 & \\
\hline 6 least deprived & 159 & 20.9 & 292 & 9.4 & 0.13 & \\
\hline \multicolumn{7}{|l|}{ Physical activity categoryc } \\
\hline I sedentary or light & 511 & 67.1 & NA & NA & & \\
\hline 2 moderate & 96 & 12.6 & NA & NA & & \\
\hline 3 moderate to vigorous & 111 & 14.6 & NA & NA & & \\
\hline 4 high & 43 & 5.7 & NA & NA & & \\
\hline
\end{tabular}

Notes: ap-values quoted are for the comparisons between categorical distributions in the two population samples; bindex of Multiple Deprivation 2004; ' based on activity thresholds given in Report of a Joint FAO/WHO/UNU Expert Consultation. ${ }^{13}$

Abbreviations: $\mathrm{CD}$, circulatory disease; NA, no data available in this category; FAO, Food and Agriculture Organization of the United Nations; WHO, World Health Organization; UNU, United Nations University. 
(variance between areas/total variance within sample) for the data shown in Figure 1 was 0.096, which indicates that a significant proportion of the variation in physical activity was explained by differences between neighborhoods.

\section{Physical inactivity and circulatory disease outcomes}

Circulatory disease events by small area-level geography Ethnicity and Index of Multiple Deprivation (IMD) ${ }^{7}$ decile variables were dropped from the analysis at the first iteration as they failed to explain a significant amount of the observed variation in the two outcome measures. The best-fit model for the prediction of circulatory disease events was:

Level 1 model

$$
\eta_{\mathrm{i} j}=\beta_{0}+\beta_{1}^{*} \text { sex }+\beta_{2}^{*} \text { quarter }+\beta_{3}^{*} \text { age decade }
$$

Level 2 model

$$
\begin{gathered}
\beta_{0}=\gamma_{00}+\gamma_{01} * \text { SPA }+u_{0} \\
\beta_{1}=\gamma_{10}+u_{1} \\
\beta_{2}=\gamma_{20}+u_{2} \\
\beta_{3}=\gamma_{30}
\end{gathered}
$$

where SPA is the standard normalized square root transformed total physical activity measure. The five fixed effects and three random effects terms in the model are summarized in Table 2.

Females were less likely than males (event rate ratio $=0.593$ ) to have a circulatory disease event. Similarly, those who were more active were less likely (event rate ratio $=0.855)$ to have a circulatory disease event. In contrast, circulatory disease events were more likely to occur in older age (decade) and later reporting quarters (UK autumn, winter months) with event rate ratios 1.578 and 1.095 , respectively.

\section{Hospital treatment costs}

Combined hospital costs of treating circulatory disease events in each output area against the mean physical activity in each area are plotted in Figure 2.

The figure shows a loess fit (a best-fit estimate from the available data using an assumed Gaussian distribution weighting and $80 \%$ of the data in each fit point $)^{14}$ of the cost per estimated head of population per area plotted against the standardized transformed mean physical activity estimated for each area. The least active areas had a mean cost per head of population of $\sim £ 200$, approximately four times that in the most active areas. The slope of the fit was steeper at lower levels of physical activity, indicating potentially greater health care cost savings by persuading the least active to become more active.

The estimated mean cost per area could be sensitive to relatively infrequent, but high-cost circulatory disease treatment events. We tested for this sensitivity using bootstrap resampling with 1,000 repeat samples from the original set of cost data per area. The mean difference between the bootstrap means was just $0.5 \%$ of the mean overall cost per area, indicating that this source of variation was not a problem in this study.

\section{Discussion \\ Physical inactivity and circulatory

\begin{tabular}{|c|c|c|c|c|}
\hline Term & Coefficient value & $\begin{array}{l}\text { Event rate ratio } \\
\text { ( } 95 \% \text { confidence interval) }\end{array}$ & Df & $P$ value \\
\hline \multicolumn{5}{|l|}{ Fixed effects } \\
\hline$\gamma_{00}$ (intercept) & -3.89 & $0.02(0.012-0.036)$ & 49 & $<0.001$ \\
\hline$\gamma_{01}(\mathrm{SPA})$ & -0.156 & $0.855(0.777-0.94 I)$ & 49 & 0.002 \\
\hline$\gamma_{10}(\operatorname{sex})$ & -0.523 & $0.593(0.467-0.752)$ & 50 & $<0.001$ \\
\hline$\gamma_{20}$ (quarter) & 0.09 & $1.095(1.04 I-1.151)$ & 50 & 0.001 \\
\hline \multirow[t]{2}{*}{$\gamma_{30}$ (age decade) } & 0.456 & $1.578(1.5-1.66)$ & 3110 & $<0.001$ \\
\hline & Standard deviation & Variance component & & \\
\hline \multicolumn{5}{|l|}{ Random effects } \\
\hline $\mathrm{u}_{0}$ (intercept) & 1.25 & 1.57 & 49 & $<0.001$ \\
\hline$u_{1}(\operatorname{sex})$ & 0.61 & 0.37 & 50 & 0.001 \\
\hline $\mathrm{u}_{2}$ (quarter) & 0.12 & 0.014 & 50 & 0.001 \\
\hline
\end{tabular} disease-related hospital events}

The relationship between physical inactivity and cardiovascular disease mortality is well established. ${ }^{15-18}$ Extending the

Table 2 Summary of best-fit model for prediction of circulatory disease events

Abbreviations: Df, degrees of freedom; SPA, standardized physical activity score. 


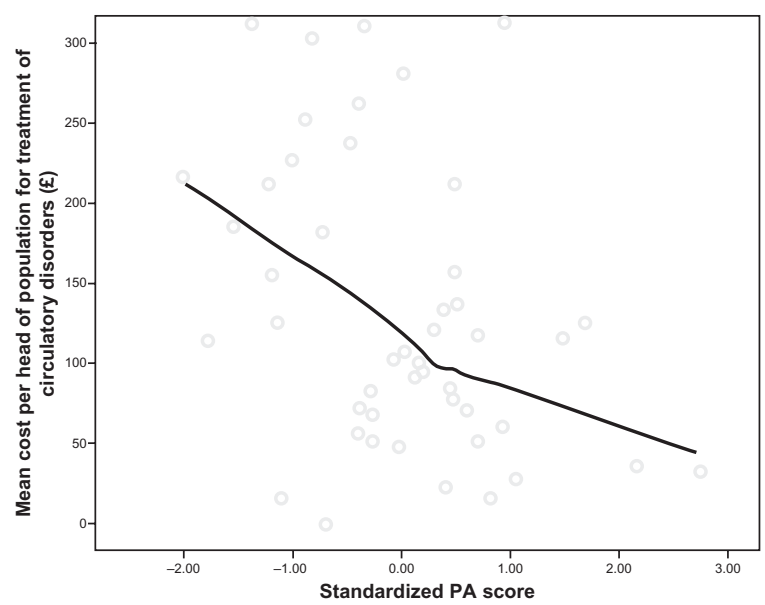

Figure 2 Mean cost per head of population per area plotted against the standardized transformed mean physical activity estimated for each area.

Note: Solid line represents a loess fit to the available data (with Gaussian weighting function and $80 \%$ of data included for each fit point).

concept of an association between exposure to physical inactivity (through the area in which you live) and increase in the risk of circulatory disease events, we hypothesized that, if such a relationship existed, then we should expect to see an increase in circulatory disease-related hospital events in areas where population physical activity was low. This relationship was confirmed by the multilevel model fitted to the data and summarized in Table 2. An increase in population physical activity level of $1 \mathrm{SD}$ unit on the physical activity measure used here could give rise to $\sim 14.5 \%$ reduction in the population risk of having a circulatory disease hospital event. One SD for the transformed physical activity measure used here was 6.4 units. Transforming back to the original physical activity measure, this equates to a difference of approximately 450 MET minutes/week at the lower end of the physical activity range and about $835 \mathrm{MET}$ minutes/week at the higher end. To put this into context, walking, moderate activity, and vigorous activity are classified as having MET intensities of 3.3, 4, and 8 respectively. Since the majority of the population may be considered to be sedentary or lightly active (Table 1), from a public health perspective, more would be gained if the least active could be persuaded to increase their physical activity by between 110 and 140 minutes of walking or moderate physical activity. This is in line with national guidelines, which recommend about 30 minutes of moderate physical activity on most days.

\section{Physical inactivity and health care costs}

The cost data for each output area in Figure 2 have been normalized by the estimated population in each area. These ranged from 177-401 with a mean of 297. The mean ages of the patient sample in each area ranged from 38-59 years with a mean of 49 years and the sex distribution also varied. We did not have reliable data on the complete age and sex distribution of the $51 \mathrm{OA}$ and so were unable to perform ageand sex-adjusted cost calculations. Nor did we consider, apart from bootstrap resampling, testing the effect of high-cost items on the mean cost per output area, the cost complexity of treatment associated with the specific circulatory disease events reported. We accept that our preliminary analysis here is limited by the available data. Nonetheless, we believe it is important to flag the issue of costs and that there may be significant cost savings to health care services if greater population levels of physical activity can be achieved. This point, again, is not new, but our data, albeit using the crude first analysis reported here, gives some indication of what savings might be feasible. Hospital episode and associated cost data are collected routinely, but rarely linked to other data sets to provide more insightful analysis. We believe that it is important from an epidemiological perspective to explore this potential more fully.

The fit to the data in Figure 2 indicates that physical inactivity may be likely to drive up health care costs for treating circulatory disease. On the other hand, a 1 SD increase in our standardized physical activity measure suggests that a mean cost reduction of $£ 35$ per head of population (assuming a linear fit) may be achieved over 2 years. Moreover, the costs for such treatments were markedly lower in areas where population physical activity was higher, raising the possibility that a preventative approach through increasing physical activity could pay for itself.

\section{Limitations}

Whilst the findings of this study are encouraging, the following limitations need to be considered. Firstly, the data are cross-sectional in nature. Thus, it is not possible to infer a causal relationship between the physical inactivity and the outcomes used. Secondly, the physical activity measures, although collected using self-report questionnaires completed by randomly sampled individuals living in the areas of interest, are considered at a population level. Conceptually, this is important as the implication is that the living environment itself may determine behaviors that contribute detrimentally to the maintenance of good long-term health. From another perspective, changes to the environment to better support physical activity may result in reduction of the health impact of chronic diseases. Thirdly, the physical activity measures gave only a short-term (in the last week) snapshot of physical activity behavior. Thus, there is a question about how 
well this snapshot captures the mean physical activity and its variation across each area and over time. This will certainly affect the robustness and generalization of the findings from the small-area study, where sample sizes ranged from 5 to 26 per area. Finally, the relatively small number of area-level units included (51) limited the number of area-level variables and possible interactions that could be included simultaneously to just one or two as well as the sensitivity to detect area-level effects.

There is also the issue of confounding through other factors that may be considered risk factors for circulatory disease, but were not captured during this research. For example, high blood pressure, high cholesterol, smoking, poor diet, and obesity are all established risk factors for circulatory disease events and must have been present to some degree within the population sampled. Thus, the constructed model should be considered relatively simple at this stage due to the nature of the available data. Similarly, our interpretation of the effects of increasing population physical activity should be considered indicative only until more sophisticated data sets are assembled and analyses are performed.

\section{Conclusion}

This study supports the use of hospital episode statistics as an outcome measure in the epidemiology of chronic disease. Other than risk factors that present at general practice, these events are the earliest indicator the health care sector receives that the patient has a chronic condition. This, therefore, makes them a potentially sensitive measure for surveillance purposes or to judge the effects of public health interventions to reduce the impact of chronic disease. Furthermore, the data are routinely collected (though not currently widely used in the context proposed here) and associated treatment costs are readily available. The geographic and temporal scales over which data are aggregated are important considerations. The former needs to be small enough in order to be able to identify where the problems are coming from (just as the Broad Street pump was identified in Snow's famous analysis of the London cholera epidemic of 1854). The latter must balance the competing requirements of being sensitive to change whilst avoiding the reliability issue associated with small sample sizes. In this analysis, increased population levels of physical activity were associated with a reduced chance of having a circulatory disease event and lower treatment costs.

\section{Acknowledgments}

The research was partially funded under the National Prevention Research Initiative, managed by the Medical
Research Council, UK (MRC Grant G0501287). The opinions expressed are those of the authors and not necessarily those of the Funding Bodies.

\section{Disclosure}

The authors report no conflicts of interest in this work.

\section{References}

1. Morris JN, Heady JA, Raffle PA, et al. Coronary heart-disease and physical activity of work. Lancet. 1953;265:1111-1120.

2. Paffenbarger RS, Hale WE. Work activity and coronary heart mortality. N Engl J Med. 1975;292:545-550.

3. Centers for Disease Control and Prevention. Physical Activity and Health: A Report of the Surgeon General. Atlanta, GA: US Department of Health and Human Services; 1996.

4. Chief Medical Officer's Report. At Least Five a Week: Evidence on the Impact of Physical Activity and its Relationship to Health. London, UK: Department of Health; 2004.

5. World Health Organization. Global strategy on Diet, Physical Activity and Health. Geneva, Switzerland: World Health Organization; 2004.

6. Warburton DER, Nicol CW, Bredin SSD. Health benefits of physical activity: the evidence. CMAJ. 2006;174:801-809.

7. Neighbourhood Renewal Unit. The English Indices of Multiple Deprivation 2004 (Revised). London, UK: Office of the Deputy Prime Minister; Apr 2004.

8. Cochrane T, Davey RC, Gidlow CJ, et al. Small area and individual level predictors of physical activity in urban communities: a multi-level study in Stoke on Trent, England. Int J Environ Res Public Health. 2009;6:654-677.

9. Speight S, Stephansen H, Clemens S. Socio-ecological Mapping of Physical Activity Behaviours and Health Outcomes in Deprived Inner-city Communities - Technical Report. London, UK: National Centre for Social Research; Dec 2007.

10. Karolinska Institute, Stockholm. International Physical Activity Questionnaire - Long version. Available from: http://www.ipaq.ki.se. Accessed July 7, 2006.

11. World Health Organization. International Statistical Classification of Diseases and Related Health Problems 10th Revision. Geneva, Switzerland: World Health Organization; 2007.

12. Raudenbush SW, Bryk AS. Hierarchical Linear Models: Applications and Data Analysis Methods, 2nd ed. Newbury Park, CA: Sage Publications; 2002:309-317.

13. Human Energy Requirements: Report of a joint FAO/WHO/UNU expert consultation. Rome, Italy: Food and Agricultural Organization of the United Nations; 2004. Table 5.1.

14. Cleveland WS, Devlin SJ. Locally weighted regression: an approach to regression analysis by local fitting. J Am Stat Assoc. 1988;83: 596-610.

15. Physical Activity Guidelines Committee. Physical Activity Guidelines Advisory Committee Report. Washington, DC: Dept of Health and Human Services; 2008.

16. Nocon M, Hiemann T, Muller-Riemenschneider F, et al. Association of physical activity with all-cause and cardiovascular mortality: a systematic review and meta-analysis. Eur J Cardiovasc Prev Rehabil. 2008;15:239-246.

17. Sofi F, Capalbo A, Cesari F, et al. Physical activity during leisure time and primary prevention of coronary heart disease: an updated metaanalysis of cohort studies. Eur J Cardiovasc Prev Rehabil. 2008;15: 247-257.

18. Shiroma EJ, Lee I-M. Physical activity and cardiovascular health: lessons learned from epidemiological studies across age, gender and race/ethnicity. Circulation. 2010;122:743-752. 


\section{Publish your work in this journal}

Clinical Epidemiology is an international, peer-reviewed, open access journal focusing on disease and drug epidemiology, identification of risk factors and screening procedures to develop optimal preventative initiatives and programs. Specific topics include: diagnosis, prognosis, treatment, screening, prevention, risk factor modification, systematic

Submit your manuscript here: http://www.dovepress.com/clinical-epidemiology-journal reviews, risk \& safety of medical interventions, epidemiology \& biostatical methods, evaluation of guidelines, translational medicine, health policies \& economic evaluations. The manuscript management system is completely online and includes a very quick and fair peer-review system, which is all easy to use. 